\title{
Resistivity studies of melt spun and annealed Al-Mn-Fe-Si multiply twinned samples
}

\author{
KULVIR SINGH, D BAHADUR and G V S SASTRY* \\ Department of Metallurgical Engineering and Materials Science, Indian Institute of \\ Technology, Powai, Bombay 400076, India \\ * Department of Metallurgical Engineering, Institute of Technology, Banaras Hindu \\ University, Varanasi 221005 , India
}

MS received 11 January 1995

\begin{abstract}
The quaternary $\mathrm{Al}_{75} \mathrm{Mn}_{10} \mathrm{Fe}_{5} \mathrm{Si}_{10}$ alloy was synthesized by induction melting, melt spinning and subsequent annealing. The melt spun sample shows the multiply twinned nodular-type microstructure. These plates surround an icosahedral seed of the nodule. Annealing induces relaxation/transformation of the seeds due to diffusion process. We studied the electrical properties of all the three states (as-cast, quenched and annealed) of $\mathrm{Al}_{75} \mathrm{Mn}_{10} \mathrm{Fe}_{5} \mathrm{Si}_{10}$ alloy. The results are discussed in the framework of Ziman-Faber theory.
\end{abstract}

Keywords. Melt spinning; multiply twinned structure; icosahedral; microstructure; electrical resistivity.

\section{Introduction}

The remarkable discovery of icosahedral phase in rapidly solidified $\mathrm{Al}_{6} \mathrm{Mn}$ alloys (Shechtman et al 1984) has generated enough interest in looking for newer systems and their physical properties (Kimura et al 1986; Wong and Poon 1986; Mizutani et al 1991). Similarly, the discovery of twinning by rotation around irrational axes in the cubic $\alpha-\mathrm{Al}_{9}(\mathrm{Mn} \mathrm{Fe})_{2} \mathrm{Si}_{2}$ phase (Bendersky et al 1989; Mandal et al 1991) marks another milestone in the field of quasicrystallography. By rapid solidification from the melt, the twin is grown in such a fashion that it retains the icosahedral motifs present in the unit cells in the same orientation across the interface, while the lattice itself undergoes a five-fold rotation around $[1, \tau, 0]$ axes. The icosahedral motif plays an important role in the growth of the twinned crystals (Goldman and Widom 1991). These irrational twins have five variants. These variants together give rise to forbidden point group symmetry which gives rise to pseudoicosahedral in multiply twinned structures. Such irrational twinning had also been observed in $\mathrm{Al}-\mathrm{Fe}-\mathrm{V}-\mathrm{Si}$ (Srivastava and Ranganathan 1992), Al-Mn-Ge (Lalla et al 1992) and Al-Mn-Cr-Si (Singh et al 1992) systems. Field and Fraser (1985) suggested that icosahedral symmetry patterns likely arose from the microtwinning among twenty twin variants of distorted diamond cubic lattice. Their twinning model is different from the present one.

It is known that all quasicrystals have complex crystalline counterparts, which are closely related to the quasicrystals in composition and local atomic structure. These are known as rational approximant structures (RAS). They have played an important role in describing the atomic-scale structure of quasicrystals, their formation, stability and physical properties (Goldman and Kelton 1993).

Usually the resistivity of quasicrystalline alloys is quite high compared to conventional crystalline alloys. But the temperature dependence of resistivity in approximant crystalline phases is similar to that in the quasicrystalline phases with a few exceptions such as 
$\mathrm{Al}-\mathrm{Cu}-\mathrm{Li}$. Al-Cu-Li quasicrystal has the negative temperature coefficient of resistivity (TCR), while the RAS of Al-Cu-Li has a positive TCR (Poon 1992; Fujiwara 1993).

In this paper we report studies on the electrical resistivity of nominal composition $\mathrm{Al}_{75} \mathrm{Mn}_{10} \mathrm{Fe}_{5} \mathrm{Si}_{10}$ for the as-cast, rapidly quenched and annealed samples.

\section{Experimental}

The quaternary alloy of nominal composition $\mathrm{Al}_{75} \mathrm{Mn}_{10} \mathrm{Fe}_{5} \mathrm{Si}_{10}$ was induction melted in argon atmosphere. Small quantities of the as-cast alloy were subsequently melt spun using a copper wheel. More details of sample preparation are given elsewhere (Mandal et al 1991). Standard four-probe DC electrical resistivity measurements using $10 \mathrm{~mA}$ current were carried out using a DRC-82C (CTI and cryosystems, USA) closed-cycle helium refrigerator in the range 8 to $300 \mathrm{~K}$. The temperature was controlled with a Lakeshore DRC-82C temperature controller to a stability of $0 \cdot 1 \mathrm{~K}$. The resistance was measured with a Datron 1071 Autocal digital multimeter. The resolution in resistance was about 1 part in $10^{5}$. Low-thermal, low-melting-point, nonsuperconducting solder was used for the contacts. Transmission electron microscopy was also done for microstructural characterization. A JEOL $200 \mathrm{CX}$ transmission electron microscope with $\pm 45^{\circ}$ tilt facility and a heating holder capable of in situ heating up to $1000^{\circ} \mathrm{C}$ was used for this purpose.

\section{Results and discussion}

The temperature dependence curves of normalized resistance of the as-cast, quenched and annealed samples of $\mathrm{Al}_{75} \mathrm{Mn}_{10} \mathrm{Fe}_{5} \mathrm{Si}_{10}$ are shown in figure 1. From this figure we

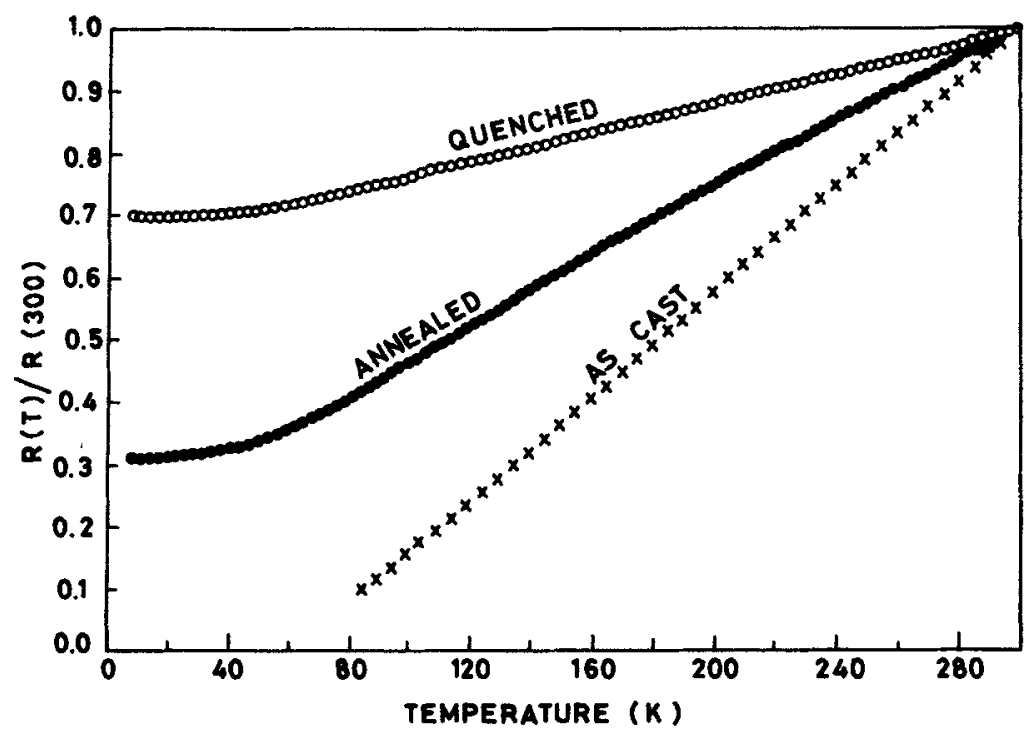

Figure 1. The normalized resistance versus temperature plot for as-cast $[R(T) / R(300 \mathrm{~K})]$, quenched and annealed $[R(T) / R(300 \mathrm{~K})] \mathrm{Al}_{75} \mathrm{Mn}_{10} \mathrm{Fe}_{5} \mathrm{Si}_{10}$ samples. 
can readily make the following observations: (i) The general nature of temperature dependence of resistance $R(T)$ and the magnitude of the resistivity for the as-cast alloys are almost similar to those of pure $\mathrm{Al}$ metal. The resistance of the as-cast alloy increases monotonically, with a resistance ratio $R(300 \mathrm{~K}) / R(80 \mathrm{~K})=11$. (ii) The melt spun (quenched) sample shows a minimum at low temperature $(\sim 20 \mathrm{~K})$, while after annealing at $400^{\circ} \mathrm{C}$ for $2 \mathrm{~h}$ it does not exhibit any minima throughout the temperature range. A saturation effect, however, is observed below $20 \mathrm{~K}$. The possibility of minima below $10 \mathrm{~K}$ cannot be ruled out. The temperature dependence of resistivity of $\mathrm{Al}-$ Mn-based quasicrystals commonly shows a minimum at low temperatures (Macko et al 1989). Similar resistivity behaviour was observed in $\mathrm{Al}_{86} \mathrm{Co}_{14}$ quasicrystal (Dunlap et al 1986). Data below the minimum are well described by root $T$ temperature behaviour predicted by the electron-electron interaction effect. Data above the minimum are usually well described by Ziman-Faber theory (Macko et al 1989). According to the generalized Ziman-Faber theory (Howson 1984) the temperature dependence of the resistivity comes from the temperature dependence of the structure factor and the Debye-Waller factor. Within this model, there can be two competing mechanisms which can in principle give rise to a resistivity minimum.

After annealing of the melt spun sample different features are observed in the $R(T)$ plot. The negative TCR which is shown by melt spun sample is not seen for annealed sample and the resistance ratio $R(300 \mathrm{~K}) / R(8 \mathrm{~K})$ also increases about two times more compared to the quenched sample. Unfortunately, we could not estimate the resistivity before and after the annealing on the same sample because of the brittle and wavy nature of samples. However, the effect on the temperature coefficient of resistivity is obvious. The above described effect in annealed sample might occur due to changes in microstructure during annealing. The multiply twinned crystals have a typical nodular microstructure as shown in figure 2 . These multiply twinned plates surround a central icosahedral seed of the nodule. After annealing, the icosahedral seeds might get transformed and relaxed due to diffusion process occurring around icosahedral seed and act as glue regions around the multiply twinned nodules. These may influence the dominant preferred electrical paths for electron transport. This effect may be greatest when such regions exist along the boundaries of the twinned nodules (Takeuchi et al 1993). In the quenched Al-Mn-Fe-Si sample annealed in situ in the microscope by R K Mandal and G V S Sastry (unpublished work), the twinned plates did not show any morphological transformation up to $600^{\circ} \mathrm{C}$. The icosahedral seed too did not show appreciable changes, though any minute changes in the icosahedral seed cannot be ruled out. Detection of such changes in the maze of the seed structure (figure 2) is difficult. Also, the relaxation of phason strains which cannot be seen as microstructural changes may be reflected in electronic properties.

The temperature dependence of resistance $R(T)$ shown in figure 1 cannot be explained by a single functional form. Hence, we have attempted here to fit the data over the limited ranges of temperature to the conventional theories applicable for the amorphous materials for want of a comprehensive model suitable for this class of materials. The obtained values for different functions are given in table 1. The quenched sample shows positive TCR in the temperature range 22 to $300 \mathrm{~K}$. We have fitted the resistivity data between 50 and $300 \mathrm{~K}$ to the relation

$$
r_{n}(T)=\frac{R(T)-R\left(T_{\min }\right)}{R\left(T_{\min }\right)}=a+b T .
$$




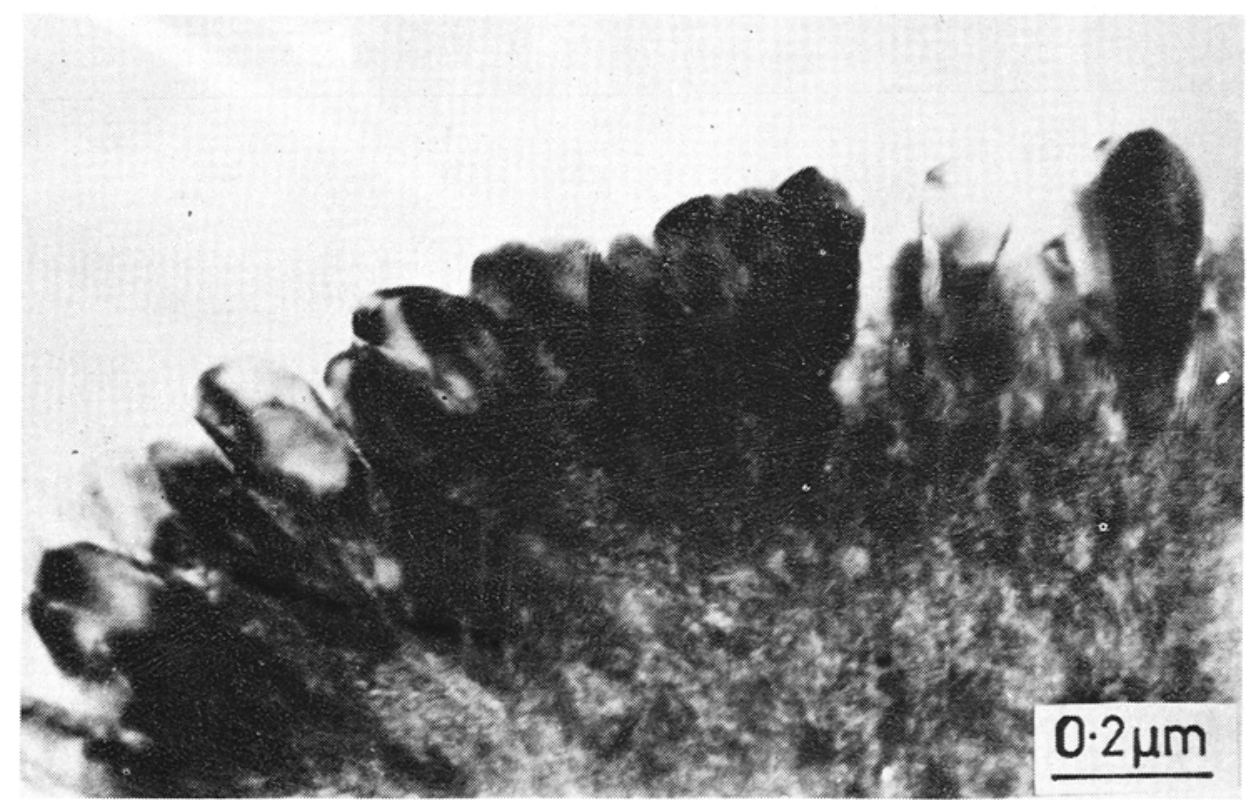

Figure 2. Transmission electron micrograph of a portion of nodule showing twin plates at the periphery and speckled contrast corresponding to the icosahedral seed at the centre.

Table 1. Fits to the normalized resistance $r_{n}(T)$ : compositions, various parameters, equations and temperature ranges of fits for quenched and annealed $A_{75} \mathrm{Mn}_{10} \mathrm{Fe}_{5} \mathrm{Si}_{10}$ are given.

\begin{tabular}{|c|c|c|c|c|c|}
\hline \multirow[b]{2}{*}{ Sample } & \multirow[b]{2}{*}{$R_{\mathrm{RT}} / R(8 \mathrm{~K})$} & \multirow[b]{2}{*}{ Functions } & \multirow{2}{*}{$\begin{array}{l}\text { Ranges of } \\
\text { temp.(K) }\end{array}$} & \multicolumn{2}{|c|}{ Parameters } \\
\hline & & & & $b\left(\mathrm{~K}^{-1}\right.$ or $\left.\mathrm{K}^{-2}\right)$ & $c\left(K^{-2}\right)$ \\
\hline $\begin{array}{l}\mathrm{Al}_{75} \mathrm{Mn}_{10} \mathrm{Fe}_{5} \mathrm{Si}_{10} \\
\text { (quenched) }\end{array}$ & 1.5 & $\begin{array}{c}a-b \sqrt{ } T \\
-a+b T^{2} \\
a+b T\end{array}$ & $\begin{array}{c}8-20 \\
35-120 \\
50-300\end{array}$ & $\begin{array}{l}6.6 \times 10^{-4} \\
9.4 \times 10^{-6} \\
1.7 \times 10^{-3}\end{array}$ & - \\
\hline $\begin{array}{l}\mathrm{Al}_{75} \mathrm{Mn}_{10} \mathrm{Fe}_{5} \mathrm{Si}_{10} \\
\text { (annealed) }\end{array}$ & $3 \cdot 1$ & $\begin{array}{l}-a+b T^{2} \\
\quad a+b T \\
a+b T-c T^{2}\end{array}$ & $\begin{array}{c}25-60 \\
50-225 \\
225-300\end{array}$ & $\begin{array}{l}4.6 \times 10^{-5} \\
9.2 \times 10^{-3} \\
1.1 \times 10^{-2}\end{array}$ & $6.3 \times 10^{-6}$ \\
\hline
\end{tabular}

In the low-temperature range 35 to $120 \mathrm{~K}$ the data are well fitted to the relation

$$
r_{n}(T)=a+b T^{2}
$$

Below $22 \mathrm{~K}$ it has a negative TCR and fits to root $T$ dependence. The electronelectron interaction is considered to be responsible for this temperature dependence at low temperature. Following Meisel and Cote (1977), the $T^{2}$ dependence arises from the temperature dependence of the structure factor using diffraction model. The high-temperature region where $T$ dependence is prevalent may be interpreted as due 
to electron-phonon interaction in the diffraction model (Altshuler and Aronov 1985; Kimura et al 1989).

In the higher temperature region between 225 and $300 \mathrm{~K}$ the annealed sample does not fit the expected relation (1). Instead, the data are fitted to an empirical relation of the form

$$
r(T)=a+b T-c T^{2},
$$

where the $T$ dependence arises from the electron-phonon interaction in the diffraction model. However, the origin of the $-T^{2}$ term is not well understood. Similar observation has been reported in case of $\mathrm{Ti}-\mathrm{Ni}-\mathrm{Fe}-\mathrm{Si}$ (Bahadur et al 1991) and in Al-Cu-Fe (Srinivas and Dunlap 1991). The data between 25 and $60 \mathrm{~K}$ fit equation (2).

Phillips (1993) has suggested that, in a two-component system consisting of icosahedral regions and non-icosahedral twinning regions, the twinning region provides the dominant electrical paths for transport. The twinning region, which is highly defective, may still be locally periodic. Almost all the background density of states $N_{\mathrm{b}}\left(E_{\mathrm{F}}\right)$ at $E=E_{\mathrm{F}}$ is associated with the twin regions in such a case and can be associated with a pseudogap which is responsible for stabilization of the structure. Following discussion on the density of states by Phillips (1993) annealing may produce an interesting feature in $N_{\mathrm{b}}\left(E_{\mathrm{F}}\right)$. For example, a minigap could develop due to relaxation through diffusion phenomena of the icosahedral seed. These minigaps in the density of states may be largely responsible for the anomalous behaviour of electrical resistivity. The $-T^{2}$ term in the high-temperature regime for the annealed sample could be due to the presence of such minigaps. Such $-T^{2}$ dependence has recently been reported in many quasicrystalline systems (Srinivas and Dunlap 1991; Singh et al 1994).

It may be concluded that multiply twinned structures not only mimic 5-3-2 symmetry but also exhibit electronic properties similar to those of the icosahedral quasicrystals. The icosahedral seeds which are randomly present presumably do not interfere with the conduction path in the as-quenched sample. However, when annealed at $400^{\circ} \mathrm{C}$, some transformation relaxation of the icosahedral seeds occurs which results in the modified resistivity behaviour explained on the basis of Phillips's (1993) hypothesis of minigaps and pseudogaps.

\section{Acknowledgements}

We are thankful to Dr Alok Banerjee for help in electrical resistivity measurements, and to Dr R K Mandal for helpful discussions. Financial support by DST, Government of India, is gratefully acknowledged.

\section{References}

Altshuler B L and Aronov A G 1985 in Electron-electron interaction in disordered systems (eds) A L Efrov and M Pollak (Amsterdam: North Holland) p. 1

Bahadur D, Das A, Singh K and Majumdar A K 1991 J. Phys. Cond. Matter 34125

Bendersky L A, Cahn J W and Gratias G 1989 Philos. Mag. B60 685

Dunlap R A, Stroink G, Dini K and Jones D F 1986 J. Phys. F16 1247 
Field R D and Fraser H I 1985 Mater. Sci. Eng. 68117

Fujiwara T 1993 J. Non-Cryst. Solids 153-154 390

Goldman A I and Kelton R F 1993 Rev. Mod. Phys. 651

Goldman A I and Widom M 1991 Annu. Rev. Phys. Chem. 42685

Howson M A 1984 J. Phys. F14 L25

Kimura K, Hasimoto T and Takeuchi S 1986 J. Phys. Soc. Jpn. 552472

Kimura K, Iwahashi H, Hasimoto T, Takeuchi S, Mizutani U, Ohashi S and Itoh G 1989 J. Phys. Soc. Jpn. 582472

Lalla N P, Tiwari R S and Srivastava O N 1992 J. Mater. Res. 753

Macko D, Hudak O and Hajko V Jr 1989 Phys. Lett. A136 327

Mandal R K, Sastry G V S, Lele S and Ranganathan S 1991 Scr. Metall. Mater. 251477

Meisel L V and Cote P J 1977 Phys. Rev. B16 2978

Mizutani U, Kaniya A, Matsuda T and Takeuchi S 1991 Mater. Sci. Eng. A133 111

Phillips J C 1993 Phys. Rev. B47 2522

Poon S J 1992 Adv. Phys. 41303

Shechtman D, Blech I, Gratias G and Cahn J W 1984 Phys. Rev. Lett. 531951

Singh Alok, Srivastava A K and Ranganathan S 1992 Proc. Symp. on Microstructure of Materials Berkeley, USA

Singh K, Bahadur D, Nigam A K, Shiv Prasad and Girish Chandra 1994 MRSI conference, Hyderabad, India

Srivastava A K and Ranganathan S 1992 Scr. Metall. Mater. 2753

Srinivas V and Dunlap R A 1991 Philos. Mag. B64 475

Takeuchi S, Akiyama H, Naito N, Shibuya T, Hasimoto H, Kadagawa K and Kimura K 1993 J. Non-Cryst. Solids 153-154 353

Wong K M and Poon S J 1986 Phys. Rev. B34 7371 